\title{
Comunidad de aprendizaje: Un proyecto potencializador de competencias profesionales en el profesorado universitario
}

\author{
Meyci De la Cruz Pérez ${ }^{1}$ \\ Anabel Calderón Menéndez ${ }^{2}$ \\ Luisbel Porvent Robirosa ${ }^{3}$
}

\section{RESUMEN}

Para el ejercicio de la profesión docente de hoy se demanda un profesorado preparado y comprometido que ejerza una educación de calidad a tono con los nuevos tiempos. Un profesorado capaz de entender la diversidad del estudiantado que enfrenta en el aula, de resolver y tomar decisiones sobre variados y complicados problemas pedagógicos, de dominar otros idiomas y las nuevas tecnologías de la información y la comunicación, esta capacidad o habilidad que tenemos los profesionales de dar solución a las dificultades que afrontamos en nuestra práctica se ha dado en llamar competencias profesionales. Para hacer frente a esas necesidades se exigen comunidades de conocimiento, aprendizaje con liderazgo, valores personales y laborales; capaces de crear, emprender, diseñar nuevas ideas y conformar grupos de trabajo, efectivos, eficaces y eficientes. Es intención de este ensayo fundamentar la comunidad de aprendizaje como proyecto educativo potencializador de competencias profesionales en el profesorado universitario.

Palabras claves: comunidad de aprendizaje, competencias.

Recibido: 11 de abril de 2017

Aceptado: 24 de noviembre de 2017

1 Doctora en Ciencias de la Educación y docente-investigadora, Filial Universitaria Municipal Majagua. Universidad Máximo Gómez Báez. Ciego de Ávila, Cuba. Correo electrónico: meyci@unica.cu

2 Docente-investigadora, Filial Universitaria Municipal Majagua. Universidad Máximo Gómez Báez. Ciego de Ávila, Cuba. Correo electrónico: anabelcm@unica.cu

3 Docente-investigadora, Filial Universitaria Municipal Majagua. Universidad Máximo Gómez Báez. Ciego de Ávila, Cuba. Correo electrónico: luisbel@unica.cu 


\title{
Learning community: A potentializing project of professional competences in university teaching staff
}

\begin{abstract}
The current teaching profession requires a prepared and committed professor who provides a high quality education as this new era demands, a professor able to understand the diversity of the students in the classroom, a professor able to solve and to make decisions about varied and complicated pedagogical issues, a professor who dominates other languages and the new technologies of information and communication, just to mention some of the dilemmas we face. The capability or ability that professionals have to solve the difficulties that we face in our practice has been called professional skills. Learning communities, learning with leadership, and personal and work values are demanded in order to meet these needs. The intention of this essay is to show learning communities as an educational project that develops professional competencies .The concept of a learning community can be defined simply as a group of people learning together using common tools in the same environment. In order to put it into practice, the context has to be transformed. All the adults who are related to the students must participate in this transformation. The groups and people have to reach a compromise, for this, they have to change the way of thinking focusing on promoting dialogue.
\end{abstract}

Keywords: community learning, skills.

\section{INTRODUCCIÓN}

Asistimos hoy en día a una realidad compleja para el ejercicio de la profesión docente, se demanda un profesorado preparado y comprometido que ejerza una educación de calidad a tono con los nuevos tiempos, un profesorado capaz de entender la diversidad del estudiantado que enfrenta en el aula, de resolver y tomar decisiones sobre variados y complicados problemas pedagógicos, de dominar otros idiomas y las nuevas tecnologías de la información y la comunicación, por sólo citar algunos de los dilemas que enfrentamos, esta capacidad o habilidad que tenemos los profesionales de dar solución a las dificultades que afrontamos en nuestra práctica se ha dado en llamar competencias profesionales.

Muchos son los autores que abordan muy acertadamente el tema de las competencias profesionales, extensa sería la lista de ellos desde las diferentes perspectivas (laboral, psicológico y curricular), un análisis integrador y muy ilustrativo resulta la definición de Jofré (2009), el cual concluye que las competencias profesionales integran simultáneamente: conocimientos, habilidades y actitudes; que se integran atendiendo a una serie de atributos personales (capacidades, características de la 
personalidad y recursos individuales); que se manifiestan a nivel de conductas y que poseen una dimensión práctica, de ejecución.

Siguiendo la idea argumental, platean Caballero et al. (2011) que el profesorado universitario que desarrolla su actividad en este escenario es consciente de la complejidad y diversidad de funciones que debe adoptar: docencia, investigación, transferencia del conocimiento, innovación y gestión, cobrando especial relevancia la promoción y consolidación de los equipos docentes, como forma de abordar la docencia y el aprendizaje en la universidad, con un doble objetivo: mejorar la calidad de la docencia y del aprendizaje de los alumnos, por una parte, y como factor de cambio de la forma en que el profesorado debe abordar sus tareas, por otro.

Por tanto, serían las instituciones educativas las responsables de ofrecer al profesorado posibilidades de formación permanente que conduzcan a construir competencias para identificar y solucionar problemas.

Propone Ávila (2008) que para hacer frente a estas necesidades, el mundo de hoy con sus avances tecnológicos, nuevas formas de relaciones interpersonales e innovación; exigen comunidades de conocimiento, aprendizaje con liderazgo, valores personales y laborales; capaces de crear, emprender, diseñar nuevas ideas y conformar grupos de trabajo, efectivos, eficaces y eficientes.

Por qué no pensar entonces, en la educación superior del futuro como un derrotero a la comunidad de aprendizaje donde puedan entenderse la universidad tradicional con las universidades virtuales y laborales, con el propósito de garantizar la superación permanente de sus profesionales.

Es intención de este ensayo fundamentar la comunidad de aprendizaje como proyecto educativo potencializador de competencias profesionales en el profesorado universitario.

\section{DESARROLLO}

\section{En torno a la definición de Comunidad de aprendizaje}

Sobre el origen de la comunidad de aprendizaje no podemos hablar de una fecha fundacional, si bien precisamos que se inició en los Estados Unidos. Por una parte, Sylvia (2001) considera a Meiklejohn como el padre del movimiento de comunidades de aprendizaje que emigró a los Estados Unidos en su niñez. Veía la educación como el medio para preparar a los estudiantes para vivir como ciudadanos responsables. Estas ideas lo llevan a fundar el Colegio Experimental, en la Universidad de Wisconsin, en 1927. En este proyecto integró sus ideas del currículo ideal el cual consistía en una comunidad de aprendizaje de dos años de duración, a tiempo completo. Por otra parte, expone Flecha (en una entrevista realizada por Vázquez, 2008) que el origen es realmente muy remoto, fue el movimiento por los derechos civiles de Estados Unidos, Malcolm X, Martin Luther King y un instituto que, precisamente se llama así, High School Martin Luther King, asesorado por la Universidad de Yale, por un grupo de profesores y profesoras afroamericanos de la Universidad de Yale, que decidieron hacer realidad en la educación el objetivo de igualdad. 
En España, otro escenario muy fuerte, el modelo educativo de las comunidades de aprendizaje se origina en Cataluña en 1978 (Flecha, 1997) en la escuela de personas adultas de la "Verneda-Sant Martí”, que está situada en un barrio obrero de Barcelona. A lo largo de varios años, el Centro de Investigación en Teorías y Prácticas Superadoras de Desigualdades (CREA), de la Universidad de Barcelona, investigó cómo desarrollar esa perspectiva de éxito educativo para todas y todos en la educación infantil, primaria y secundaria. Así llegó a elaborar el modelo de comunidades de aprendizaje que, como centro público de investigación, ofreció desinteresadamente a quienes quisieran llevarlo a cabo.

Hoy en día diversos centros funcionan con esta metodología en distintas comunidades autónomas como Andalucía, Aragón, Castilla la Mancha, Cataluña, Madrid, Extremadura y País Vasco, en las cuales colaboran personas y equipos de otras universidades españolas. También dos países sudamericanos han comenzado a trabajar en esta línea, Brasil, Chile y sigue extendiéndose por otros países.

Revisando definiciones sobre comunidad de aprendizaje encontramos algunas bien interesantes, las cuales relacionamos:

Este tipo de organización, según Astin (1985), construye un sentido de identidad de grupo, de cohesión, continuidad y de diversas experiencias curriculares y cocurriculares que fomentan la integración de los estudiantes.

Castillo (1995) señala que una comunidad de aprendizaje es donde las personas de todos los niveles están mejorando continua y colectivamente su capacidad para crear las cosas que quieren crear. El proceso de aprendizaje es un proceso que ocurre con el tiempo y en el que cambian las creencias, las maneras de ver el mundo, las destrezas y las capacidades del estudiante.

Plantea Torres (2004) que una comunidad de aprendizaje es una comunidad humana organizada que construye y se involucra en un proyecto educativo y cultural propio, para educarse a sí misma, a sus niños, jóvenes y adultos, en el marco de un esfuerzo endógeno, cooperativo y solidario, basado en un diagnóstico no sólo de sus carencias sino, sobre todo, de sus fortalezas para superar tales debilidades.

Según Vallaeys (2006) cuando se consulta la bibliografía sobre el tema, se encuentra en general tres tipos de definición de la comunidad de aprendizaje:

1. En relación con la educación formal: una comunidad de aprendizaje es el resultado de una decisión, por parte de una institución educativa, de abrir sus puertas hacia su entorno social, hacia la comunidad, para crear nuevas dinámicas de aprendizaje con participación de actores externos.En relación con la educación informal: una comunidad de aprendizaje procede de la decisión de una comunidad social de reunirse para autoeducarse, compartiendo recursos y 
habilidades entre todos para que todos puedan desarrollar sus capacidades.

2. En relación con la educación virtual: un grupo de personas usa tecnologías de comunicación para crear sinergia y conocimientos, a través de un proceso de investigación compartida en un espacio virtual.

Existen muchos autores, estudiosos, investigadores y así mismo sus supuestos sobre la comunidad de aprendizaje, no constituye nuestro objetivo realizar un análisis minucioso de qué es la comunidad de aprendizaje; aunque, existen ciertos elementos comunes en las definiciones que identificamos y que para el propósito de este artículo podemos mencionar: la responsabilidad es compartida, es decir, todos los miembros de la comunidad son participes y decisores en el proceso de aprendizaje, por lo que este es un proceso dialógico, activo y colaborativo; el conocimiento se entiende como dinámico, adquirirlo no supone reproducirlo sino construirlo a partir de una comprensión propia.

\section{Comunidad de aprendizaje. Fundamentos del proyecto educativo}

Vamos a comenzar con lo señalado por Torres (2004) sobre la comunidad de aprendizaje y que sin dudas demuestra las posibilidades de su práctica en diferentes realidades sociales y educativas, todo ello, constituye muy sintéticamente el sustrato de los fundamentos de la comunidad de aprendizaje como proyecto educativo,

La autora expresa que la comunidad de aprendizaje:

- Parte de la condición de que toda comunidad humana posee recursos, agentes, instituciones y redes de aprendizaje, que son necesarias identificarlas, valorarlas, desarrollarlas y articularlas con el fin de construir un proyecto educativo y cultural que parta de las propias necesidades y posibilidades de sus actores.

- Adopta una visión amplia de lo educativo, abarcando diversos ámbitos de aprendizaje: la familia, el sistema escolar, la comunidad, la naturaleza, la calle, los medios de comunicación, la iglesia, el lugar de trabajo, el club, la biblioteca, la cancha deportiva, el huerto, el patio escolar, el cine, el teatro, el museo, la granja, el zoológico, el circo, etc. De este modo se articulan la educación escolar y la educación extraescolar, la educación formal y la informal.

- Asume como objetivo y como eje el aprendizaje más que la educación. Pretende satisfacer necesidades básicas de aprendizaje de la comunidad, identificando dichas necesidades, así como los espacios y maneras más apropiados para satisfacerlas en cada caso. Da gran importancia a los aspectos pedagógicos y a la renovación pedagógica en los distintos ámbitos de enseñanza y aprendizaje.

- Se basa en la premisa de que únicamente un esfuerzo conjunto entre todos los agentes comunitarios y el aprovechamiento de todos los recursos humanos y materiales disponibles en cada comunidad, pueden hacer posible la educación para todos y el aprendizaje permanente. Toma la necesidad del diálogo, la alianza y la concertación de actores diversos en torno a un 
proyecto educativo y cultural compartido.

- Estimula la búsqueda y el respeto por lo diverso, al reconocer que cada grupo y comunidad tiene recursos, necesidades y realidades específicas, lo que determina proyectos educativos y culturales también específicos, ajustados a cada realidad y contexto. Se promueve la construcción y experimentación de experiencias diversas, con capacidad para inspirar a otros, más que para ser replicadas.

- Busca mostrar la importancia y el potencial que tiene el desarrollo de sistemas de aprendizaje generados y desarrollados a nivel local, basados en la cooperación, la solidaridad, el aprendizaje intergeneracional y la sinergia de esfuerzos y contando con un sistema organizado de apoyos a nivel intermedio y central.

- Se propone como un modelo de desarrollo y cambio educativo de abajo hacia arriba y de adentro hacia fuera, capaz de influenciar los modos tradicionales de pensar.

En entrevista realizada por Manuel Vázquez en el 2008 al catedrático en Sociología de la Universidad de Barcelona, Ramón Flecha, señalaba que la comunidad de aprendizaje como modelo educativo tiene tres puntos fuertes: uno de los puntos más fuertes es que logra mejores resultados; es decir, logra mejores resultados medibles en evaluaciones externas. Otro punto fuerte es la implicación de la comunidad: la comunidad se implica porque ve que el centro está mejorando; porque ve que, por fin, se le quiere dar un buen servicio y entonces se involucra. Y el tercer punto fuerte es que hay una base científica de la comunidad científica internacional.

Así mimo se plantea la crítica más marcada a este modelo que es el eclecticismo metodológico, lo cual constituye una debilidad metodológica, es decir, no tiene una opción metodológica concreta, en opinión de Flecha (2008) esto más que una limitación establece una riqueza porque las comunidades de aprendizaje se pueden hacer con libros de texto muy tradicionales, sin libros de texto, se pueden hacer con muchas ópticas metodológicas porque es para todos, a lo cual añadimos que en su diversidad radica su fortaleza.

También podemos agregar como argumento de la comunidad de aprendizaje como proyecto educativo lo apuntado por Vallaeys (2006) en relación a la variedad de los tipos de comunidad de aprendizaje y las temáticas encontradas en ella lo cual resulta extenso; además se pueden abordar de distintas maneras y con diferentes actores:

- Pasar un convenio con una comunidad local (urbana o rural).

- Tratar un problema de desarrollo de modo temático y global (por ejemplo: el agua) y pasar convenios con diferentes actores a medida que se van diseñando las acciones por realizar.

- Crear una comunidad de aprendizaje dentro de la universidad para resolver un problema de esta (por ejemplo: el impacto ambiental, formación del profesorado).

- Asociarse en red con otras universidades para tratar un problema global.

- Se puede crear sinergia entre diferentes actores externos que no siempre suelen asociarse, 
aprovechando la confianza que puede generar una entidad académica.

- Se pueden articular actores internos diferentes que no suelen trabajar juntos: profesorado de diversas especialidades, investigadores y estudiantado, profesorado y administrativos, estudiantado y administrativos, etc.

En nuestra modesta opinión las comunidades de aprendizaje brindan la posibilidad de formación a los profesionales que quieren seguir superándose en su práctica educativa, pues es un modelo de formación abierto, participativo y flexible que se fortalece cuando sus miembros son capaces de:

\section{Dialogar reflexivamente sobre su práctica educativa}

Los miembros de una comunidad de aprendizaje hablan sobre su situación y sobre los retos específicos que enfrentan. Juntos, desarrollan un conjunto de normas, creencias y valores comunes que conforman una base para la acción. Los miembros de una comunidad de aprendizaje pueden utilizar estas discusiones para criticarse ellos mismos, al igual que al programa de aprendizaje dentro del cual trabajan. Estas críticas pueden tomar diferentes direcciones: pueden enfocarse en un currículo apropiado y en cómo presentárselo a los estudiantes que están aprendiendo, en una asistencia técnica efectiva o en estrategias de entrenamiento, en desarrollo, en formas de mejorar el ambiente de aprendizaje o el clima del aula.

\section{Compartir y aprender de su práctica pública}

Los miembros comparten, observan y discuten los métodos de entrenamiento, así como su filosofía, un método de colaboración entre compañeros, que no juzga ni evalúa, sino que se ayudan y comparten experiencias. Cuando comparten la práctica públicamente, los practicantes aprenden nuevas formas de hablar sobre lo que ellos hacen y las discusiones hacen surgir nuevas relaciones entre los participantes.

\section{Concentrar sus esfuerzos en el mejoramiento de la calidad.}

Los practicantes se concentran en mejorar la calidad de la realidad educativa. Asumen que todos sus miembros pueden aprender y cambiar. Dentro de una fuerte comunidad de aprendizaje, este enfoque es una obligación sentida por todos los practicantes.

\section{Colaborar}

Una comunidad de aprendizaje anima a los practicantes a trabajar juntos, no sólo para desarrollar una comprensión compartida de sus prácticas, sino también para producir materiales y actividades que mejoren las condiciones de los programas tanto para el profesorado como para el estudiantado y el desarrollo de competencias profesionales.

\section{Compartir normas y valores}

A través de sus palabras y acciones, los practicantes reunidos en una comunidad de aprendizaje reafirman sus valores comunes respecto a importantes asuntos referentes a la calidad de su realidad. Estos valores pueden atender a la calidad de las experiencias que día a día se ofrece al estudiantado, 
a los esfuerzos para apoyar el desarrollo profesional del profesorado y a la forma de hacer que la comunidad participe efectivamente.

\section{CONCLUSIONES}

La comunidad de aprendizaje es un proyecto educativo que potencializa las capacidades individuales y sociales de todos y cada uno de sus integrantes. Su importancia radica en trazarse metas, tener una visión prospectiva para dimensionar el desarrollo armónico y social de las comunidades, municipios, barrios, escuelas, aulas, entre otros. $\mathrm{Su}$ concepto se extiende a todos los ámbitos sociales, educativos, políticos, económicos, de desarrollo humano, de comunicación, y la premisa fundamental reside en fortalecer los aspectos básicos de todos los seres humanos: crecimiento cultural, físico, psicológico, interpersonal, cognoscitivo. Por lo tanto, la comunidad de aprendizaje es una comunidad humana, territorialmente delimitada, que asume un proyecto educativo y cultural, en donde niños, jóvenes y adultos se comprometen con el aprendizaje, en ella la responsabilidad es compartida. El conocimiento se entiende como dinámico, adquirirlo no es una lista de elementos a reproducir en un examen, sino construir una comprensión propia de la materia.

El concepto de comunidad de aprendizaje puede ser definido de forma sencilla como un grupo de personas que aprende en común, utilizando herramientas comunes en un mismo entorno, no obstante condiciones mínimas que deben darse para que existan, se hacen necesarios cambios institucionales que faciliten el desarrollo de dichas comunidades, deben buscarse modelos efectivos para su funcionamiento, avanzar en las innovaciones técnicas necesarias para permitir que aparezcan y facilitar herramientas de trabajo que propicien entornos modernos y flexibles, la participación de sus miembros debe ser abierta y de forma horizontal, todos deben acceder en igualdad de condiciones sin tener en cuenta su condición o el lugar donde habiten, el trabajo debe ser colaborativo por grupos y debe situarse al alumnado en el centro de los objetivos del aprendizaje.

Para que se pongan en práctica hay que transformar el contexto, todas las personas adultas que se relacionan con el estudiantado deben participar en esa transformación. Los colectivos y personas han de llegar a un compromiso, para ello, se han de cambiar la forma de pensar centrándose en promover el diálogo.

\section{REFERENCIAS BIBLIOGRÁFICAS}

Astin, A. (1985). Achieving educational excellence. San Francisco: Jossey- Bass.

Ávila, M. (2008).Modelo de comunidad de aprendizaje: tecnología de información y comunicación como estrategia de desarrollo local. Recuperado de http://www.monografía.com

Caballero, K.; Hernández, A. M.; Herrera, L. y Trujillo, J. M. (2011). Competencias docentes del profesorado novel participante en un proyecto de mentorización: Implicaciones para el 
desarrollo profesional universitario. Revista de curriculum y formación del profesorado. 15 (3): 214-241.

Castillo, A. (1995). Visión de la escuela intermedia como comunidad de aprendizaje. (Ponencia presentada en la Conferencia Anual de la Iniciativa para la Renovación de la Escuela Intermedia).

Flecha, R. (1997). Compartiendo palabras. El aprendizaje de las personas adultas a través del diálogo. Barcelona: Paidós.

Jofré, G. (2009). Competencias profesionales de los docentes de enseñanza media de Chile: Un análisis desde las percepciones de los implicados. (Tesis doctoral). Universidad de Barcelona, España.

Sylvia, M. (2001). Creando comunidades de aprendizaje a nivel universitario.

Torres, M. A. (2004). Comunidad de aprendizaje: Repensando lo educativo desde El desarrollo local. (Simposio Internacional sobre Comunidades de Aprendizaje). Barcelona, España.

Vallaeys, F. (2006). Comunidades de aprendizaje para el desarrollo. (CD-ROM) Iniciativa Interamericana de Capital Social, Ética y Desarrollo del BID. Chile.

Vázquez, M. (2008). Comunidad de aprendizaje e inclusión: Entrevista a Ramón Flecha. P@KEN-REDES Revista Digita. 1(3). Centro del Profesorado de Alcalá de Guadaíra. 